\title{
Synchrotron X-Rays for Microstructural Investigations of Advanced Reactor Materials
}

\author{
WOLFGANG HOFFELNER, ANNICK FROIDEVAL, MANUEL POUCHON, \\ JIACHAO CHEN, and MARIA SAMARAS
}

\begin{abstract}
X-rays from synchrotron beamlines provide a powerful tool for materials analysis in circumstances where long-term materials degradation under complex loading conditions (e.g., temperature, irradiation, and stress) becomes important. This may occur for advanced gas cooled reactors. Synchrotron X-rays can help to improve lifetime assessments by providing a more indepth understanding of microstructural damage. This article summarizes results of X-ray absorption fine spectrum analysis and X-ray magnetic circular dichroism synchrotron techniques. They were employed to evaluate various microstructural features, which are important in understanding the lifetime of materials exposed to extreme conditions. Dispersoid strengthening by yttria particles, conditions that produce nanocrystal Zircaloy, and the role of magnetism on the stability of ferritic steels were taken as examples.
\end{abstract}

DOI: $10.1007 / \mathrm{s} 11661-007-9326-\mathrm{z}$

(C) The Minerals, Metals \& Materials Society and ASM International 2007

\section{INTRODUCTION}

ADVANCED future nuclear (Generation IV) fission and fusion plants require materials that sustain extreme service conditions, such as temperatures of up to 1000 ${ }^{\circ} \mathrm{C}$, irradiation damage of more than 100 displacement per atom (dpa), and hostile environments such as impure helium, sodium, liquid metal, or water. ${ }^{[1]}$ These conditions can cause thermally- or irradiation-induced microstructural changes and defects in the materials. Degradation of the materials due to static loads (creep) and alternating loads (fatigue) must also be taken into account. An additional challenge concerns the lifetime of some components, such as pressure vessels or core internals, where 60 years of service are envisaged. Methods for damage assessment under these conditions are required not only for established materials, but also for newer materials that have inadequate long-term assessment databases. A powerful methodology to predict and design materials and their behavior is the multiscale approach, which combines mechanical testing of sample sizes ranging from large scale to subsized complemented with synergistic multiscale modeling. ${ }^{[2]}$ Although failure of a component is usually considered as a macroscopic event, the main portion of damage during exposure time occurs on a microscopic and even nanoscopic level. The determination of the dependence of

WOLFGANG HOFFELNER, Project Manager, and ANNICK FROIDEVAL, MANUEL POUCHON, JIACHAO CHEN, and MARIA SAMARAS, Scientists, are with the Paul Scherrer Institute, Nuclear Energy and Safety Department, CH-5232 Villigen PSI, Switzerland. Contact e-mail: wolfgang.hoffelner@psi.ch

This article is based on a presentation given in the symposium entitled "Materials Issues for Advanced Nuclear Systems," which occurred February 25-March 1, 2007 during the TMS Annual Meeting in Orlando, Florida under the auspices of the Corrosion and Environmental Effects Committee of ASM-TMS.

Article published online September 27, 2007 local mechanical properties from the microstructure is therefore of utmost importance. X-ray beamline techniques provide very powerful tools for analyzing material damage at the microscopic level, which are complementary to the well-established techniques of scanning electron microscopy (SEM) and transmission electron microscopy (TEM). Table I lists the main properties of advanced X-ray beamline techniques relevant to damage characterization. Reliable modeling of materials necessitates experimental validation. Comparison must be done at the microstructural level, indicating the significance of using the microstructural techniques available at synchrotron irradiation facilities in this field.

Extended X-ray absorption fine structure (EXAFS) and X-ray magnetic circular dichroism (XMCD) coupled with photoemission electron microscopy (PEEM) are used as examples of synchrotron techniques to demonstrate their application to advanced microstructural analysis. The work presented in this article concerns three materials that are currently under worldwide investigation for advanced fission applications: an oxide dispersion strengthened (ODS) ferritic steel (PM2000), a zirconium alloy (Zircaloy), and binary $\mathrm{Fe}-\mathrm{Cr}$ alloys, which are the basis of many ferritic steels.

\section{EXPERIMENTAL DETAILS}

\section{A. Materials}

The ODS steels are attractive candidate materials for several advanced nuclear applications. They are particularly important for high-temperature applications, where creep of conventional steels becomes an issue. The investigated ODS material is a commercial grade PM2000 plate received from Plansee (Plansee SE, Reutte/Austria). The chemical composition is shown in Table II. It was manufactured by mechanical alloying in a high-energy mill to produce a solid solution that 
Table I. Characteristics of X-Ray Beamlines with Respect to Damage Analyses

\begin{tabular}{ll}
\hline Beamline & \multicolumn{1}{c}{ Consequences } \\
\hline High intensity & $\begin{array}{l}\text { in-situ tests are possible } \\
\text { small specimen area } \\
\text { Focused beam }\end{array}$ \\
Selective & $\begin{array}{l}\text { another one } \\
\text { broad range of photon } / \text { matter } \\
\text { interactions } \\
\text { Flexible with respect to }\end{array}$ \\
wavelengths & analyzed \\
Short pulses & spin and magnetism \\
Polarization &
\end{tabular}

contains a uniform dispersion of yttria. Hot isostatic pressing followed by a hot- and cold-rolling procedure consolidated the powder. Finally, the material was annealed. A statistical analysis of size and distribution of the dispersoids of the samples has been given in another article. ${ }^{[3]}$ The material was investigated in the as-received condition and after $\mathrm{He}$ ion implantation to study the stability of the dispersoids under irradiation. Details are described in References 4 and 5.

Zircaloys are very well-established cladding alloys. They are usually employed as pipes containing fuel tablets and are made with a clearly textured polycrystalline microstructure. The improved mechanical properties of nanocrystalline Zircaloy, the emphasis of this work, are of interest for eventual applications in the supercritical water reactor (SCWR). The nanograined Zircaloy was obtained by massive plastic deformation (10 step cold rolling) at room temperature. Experiments were performed with the qualities Zircaloy 2 and Zircaloy 4 (chemical analyses are given in Table II). Small slabs with typical sizes of $20-\mathrm{mm}$ length, $5-\mathrm{mm}$ width, and $0.3-\mathrm{mm}$ height were cold rolled creating deformations from 20 to 200 pct. The resulting microstructure was analyzed in the TEM.

Ferritic steels such as pressure vessel steels, low alloy steels, and ODS steels (e.g., PM2000), are composed of $\mathrm{Fe}$ with various concentrations of $\mathrm{Cr}$ in the 0 to $20 \mathrm{wt}$ pet $\mathrm{Cr}$ range. Modeling of the $\mathrm{Fe}-\mathrm{Cr}$ alloy is currently an active field which needs one to one experimental validation, leading to the use of synchrotron techniques to perform such comparisons. These comparisons enable validation of modeling techniques. In this case, they were used to investigate the chosen interatomic potentials and to deepen the understanding of the effect of magnetism on the structure and stability of the material as a function of $\mathrm{Cr}$ content. Two button melts of $\mathrm{Fe}-\mathrm{Cr}$ alloys were prepared by arc-melting stoichiometric proportions of pure metallic $\mathrm{Fe}$ and $\mathrm{Cr}$ under an argon atmosphere with previous vacuum purging. The composition of the $\mathrm{Fe}-\mathrm{Cr}$ alloys was determined by inductively coupled plasma-atomic emission spectrometry (ICP-AES). The chromium content of the alloys was measured to be 5.8 and $11.9 \mathrm{wt}$ pct. The chemical analysis can be seen in Table II. Prior to the PEEMXMCD measurements, the two alloys were cut as flat samples of 7-mm length, 7-mm width, and 1-mm thick. They were polished down to $250 \mathrm{~nm}$ and magnetized up to their saturation of the remanent magnetization in a magnetic field of 1300 Oe.

\section{B. X-Ray Analyses}

\section{Extended $X$-ray absorption fine structure}

The spectroscopic technique of EXAFS allows characterization of the local atomic structure of any material. Interatomic distances, as well as the nature and number of neighboring atoms located around the central absorbing atom, are the main results from EXAFS analyses. During the photoelectric effect, which is the predominant process of X-ray absorption for photon energies below $100 \mathrm{keV}$, an inner-shell electron is ejected. This is called the photoelectron and is represented as an outgoing spherical wave centered at the emitting atom. This electron wave is scattered by the neighboring atoms, and the new waves emanating from each scattering site are superimposed to the initial outgoing wave. The interferences appear as oscillations in the absorption spectrum. These oscillations are superimposed to the atomic portion of the absorption, which is a smooth signal, monotonically decreasing with energy. The modulations represent the EXAFS signal defined as $\chi(\mathbf{k})$, which are the sum of individual waves from different types of neighboring atoms located in different shells. By Fourier transformation of the measured EXAFS signal, the spatial distribution of the individual atom shells around the absorbing atom can be quantitatively determined. The EXAFS technique is sensitive to aperiodic local lattice distortions (10 pct of the atoms must be involved for detection) that are invisible to conventional diffraction-based crystallographic methods. The technique of EXAFS therefore provides short-range information that is complementary to the long-range average structures obtained through diffraction analysis.

Table II. Chemical Compositions of the Materials Investigated (Weight Percent)

\begin{tabular}{|c|c|c|c|c|c|c|c|c|c|}
\hline Material & $\mathrm{Al}$ & $\mathrm{Cr}$ & $\mathrm{Fe}$ & $\mathrm{Ni}$ & $\mathrm{Sn}$ & $\mathrm{Ti}$ & $\mathrm{Y}_{2} \mathrm{O}_{3}$ & $\mathrm{Zr}$ & Remarks* \\
\hline $\mathrm{Fe} 6 \mathrm{Cr}$ & 0.005 & 5.83 & 94.2 & - & - & 0.006 & - & - & 1 \\
\hline $\mathrm{Fe} 12 \mathrm{Cr}$ & 0.004 & 11.9 & 88.1 & - & - & 0.006 & - & - & 1 \\
\hline PM2000 & 5.5 & 20 & 73.5 & - & - & 0.5 & 0.5 & - & 2 \\
\hline Zircaloy 2 & - & 0.1 & 0.17 & 0.05 & 1.35 & - & - & 98.31 & 1 \\
\hline Zircaloy 4 & - & 0.07 to 0.13 & 0.19 to 0.24 & 0.007 & 1.2 to 1.7 & - & 一 & bal & 2 \\
\hline
\end{tabular}

* Remarks: 1 represents in-house analysis, and 2 represents supplier data. 


\section{PEEM and $X M C D$}

Nanomagnetic properties of a material can be analyzed by means of PEEM with circularly polarized soft X-rays, which allows performance of soft XMCD measurements. Due to the element specificity of XMCD, it is possible to individually probe each element of the sample and to obtain electronic and magnetic properties. This technique requires a high brilliance source of circularly and linearly polarized X-rays available in third generation synchrotrons. This technique combines magnetic spectroscopy and microscopy and is called imaging magnetic microspectroscopy. It allows imaging of the surface magnetic domain microstructures of materials by using left- and right-handed circularly polarized synchrotron radiation.

Figure 1 shows a scheme of the PEEM measuring device. The degree of circular polarization and the grazing incident angle between the incoming beam and the sample surface were set to $75 \mathrm{pct}$ and $25 \mathrm{deg}$, respectively. The measurements were performed at the $\mathrm{FeL}_{2,3}$ absorption edges using left- and right-handed circularly polarized synchrotron radiation emitted from the WERA beamline, ANKA (Karlsruhe, Germany). The ferromagnetic domains were measured in a zero magnetic field at room temperature.

\section{RESULTS AND DISCUSSION}

\section{A. Microstructural Investigations}

\section{EXAFS with an Irradiated ODS Alloy}

A selective investigation of the structure of the yttria particles was possible with EXAFS, although the particles were only present in a very small fraction. The He-induced radiation damage has been found to be one dpa in the ferritic matrix. About one third of this radiation damage was determined for the yttria particles. ${ }^{[4]}$ The damaged surface layer on the sample is very shallow, with about one third of the EXAFS signal in

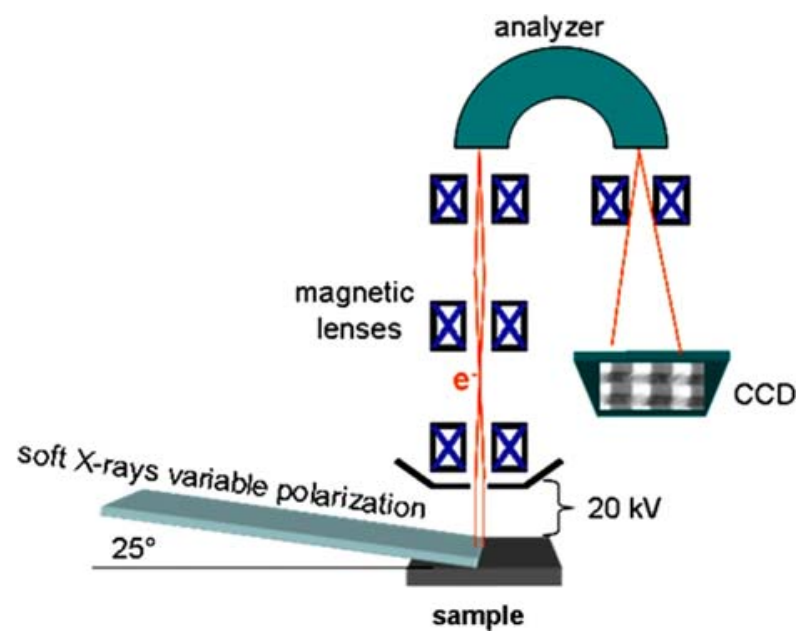

Fig. 1-Scheme of the PEEM, where CCD is the charged coupled device camera (source: F. Nolting PSI). fluorescent mode derived from this zone. This change is, however, enough to show that a major change in the yttria particle structure would be visible by the EXAFS signal.

Figure 2 depicts the Fourier transformed data of the yttrium measurements on the irradiated and the nonirradiated samples. For the transformation, a sine window ranging from roughly $\mathrm{k}=2$ to $13 \AA^{-1}$ was chosen, and no phase correction was performed. The first peak corresponds exactly for all measurements. It is attributed to an $\mathrm{O}$ shell around the absorbing yttrium atom (the FEFF simulation in Reference 4). The succeeding shells are very similar, not showing any tendency as a function of the position. The comparison demonstrates that the distribution is the same in both cases. For shell radii $R>6.5 \AA$, small differences are visible due to the fact that the reference sample was measured at low temperature. The amplitude of the first peak located at $1.5 \AA$ displays the same intensity in both cases. By contrast, the other peaks of the Fourier transformation exhibit different amplitudes, which may be due to a difference in the coordination number and which would generally be smaller in the case of an irradiated sample. The difference is, however, too small to conclude a real difference in the structure. It is important to note that the differences in amplitude might partially be induced by the temperatures at which the experiments were conducted. Up to the tested radiation dose, the yttria particles contained as dispersions within PM2000, prove to have good radiation resistance. The $0.33 \mathrm{dpa}$ displacement damage, and the 4000 atomic ppm (appm). He introduced into the particles, do not change the

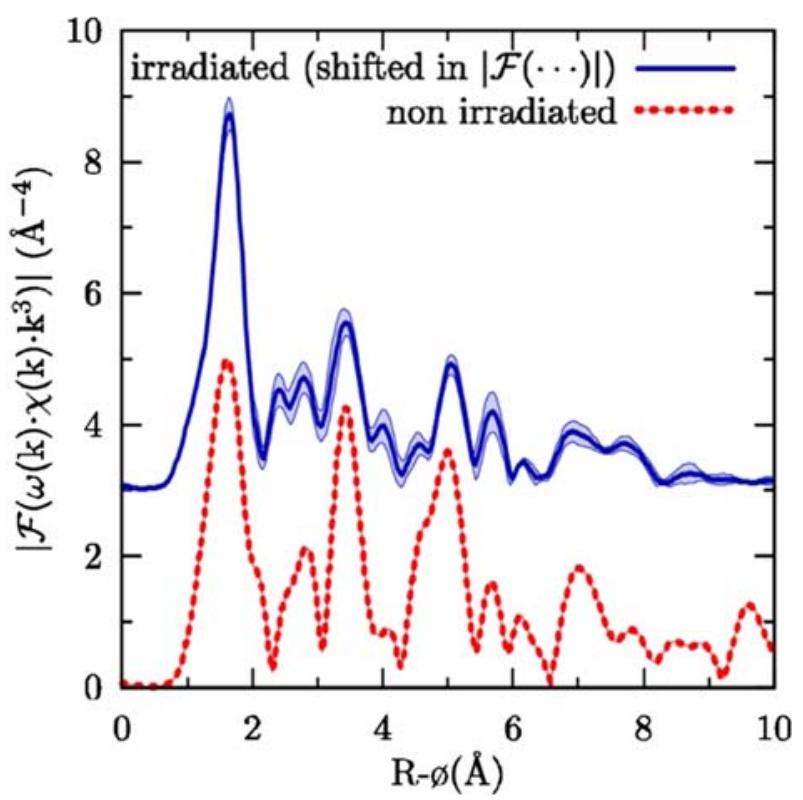

Fig. 2-Fourier transforms of yttrium EXAFS signals $\chi(\mathbf{k})$ (multiplied with the cube of the wave vector $\mathbf{k}$ ) for irradiated (upper signal presented with an offset of $3 \AA^{-4}$ for better distinction) and unirradiated (lower signal) sample. $\omega(\mathbf{k})$ is the transformation window. Radial distribution function of irradiated sample is an averaged curve from five measured locations, including two error lines, which represent the standard deviation. 
structure of yttria, although the surrounding matrix is clearly damaged. ${ }^{[6]}$ As no significant effect was detected, this indicates that the particles remain stable. With their important role as pinning points for dislocations, and with their stability under radiation condition, these results indicate that the yttria ODS particles should be able to maintain the material creep properties and confirm the importance of their inclusion to extend the lifetime of these candidate materials. This is an important finding with respect to very high-temperature reactor (VHTR) applications where only low to moderate irradiation damage is expected during service. Higher irradiation doses and $\mathrm{He}$ trapping by dispersoids, typical for fusion and for fast fission reactors, are expected to show effects in the EXAFS spectra according to first TEM results. This will be investigated in the future with a shielded test device.

\section{EXAFS of Nanograined Zircaloy}

An earlier EXAFS investigation of cold rolled Zircaloy ${ }^{[7]}$ revealed deformation-induced changes in the numbers of next neighbors in different shells without detailed correlation with microstructure. Besides changes in the range of 0 to 20 pct cold deformation, a significant increase in the number of next neighbors in the third shell at very high deformations (Figure 3) appeared. The present investigations were aimed at analyzing the microstructure developing during very high ( $>50 \mathrm{pct}$ ) deformations. The EXAFS response to microstructures developing at deformations below 50 pct was not further investigated here. It is expected that it can be correlated with well-known deformation effects in Zircaloy, such as texture formation and twinning. A review of these mechanisms can be found in Reference 8. In this research, we therefore investigated the sample deformed to $100 \mathrm{pct}$ in the TEM where a pronounced nanograined structure became visible. This nanograined structure led to the performance of additional cold deformation tests with Zircaloy 2 and 4 . They revealed that after approximately 50 pct cold rolling deformation, the first clusters of nanograins appear. A detailed study of the formation mechanism of

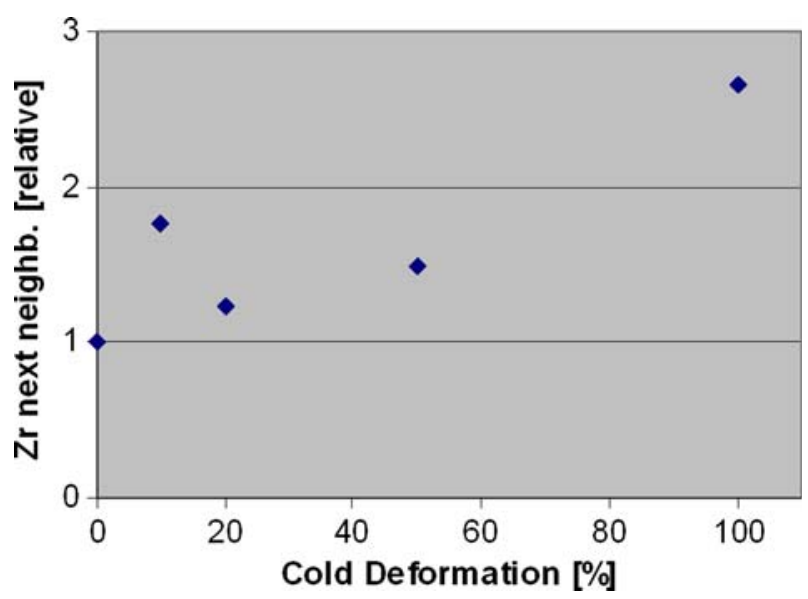

Fig. 3-Relative change of next neighbors in the third shell in Zircaloy determined by EXAFS analysis (data replotted from Ref. 7).

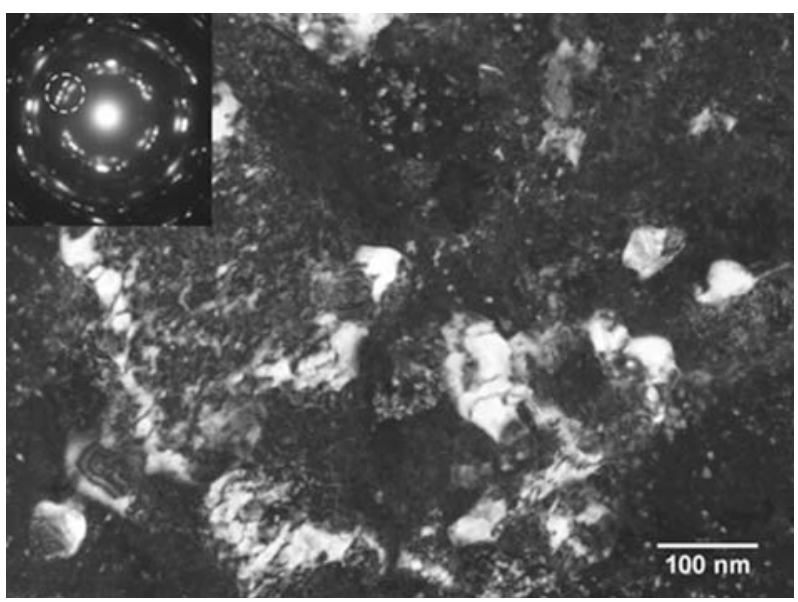

Fig. 4-Zircaloy 4 recrystallized after 100 pet deformation by cold rolling. Developed nanocrystals are clearly visible (TEM dark-field micrograph).

nanograins is currently under investigation. It is likely that randomly oriented nanograins are a result of breakdown of submicronic polygonal grains. This multistep mechanism was published for $\alpha$-titanium by $\mathrm{Zhu}$ et al. ${ }^{[9]}$ The number of these clusters increases with increasing deformation and at about 150 pct the entire sample becomes nanograined. A TEM dark-field image of a sample deformed to 100 pct is shown in Figure 4. It is therefore very likely to assume that the changes in the third shell next neighbors correspond to the development of a pronounced nanostructure. Based on these findings, in-situ EXAFS tests for the investigation of the thermal stability of this nanostructure as well as tests with irradiated nanostructures will be performed. The results show that $50 \mathrm{pct}$ deformation enables the formation of nanograined clusters and that by increasing the deformation the number of clusters increased until at about 150 pct, where a pure nanostructure was obtained.

\section{B. PEEM-XMCD of Binary Fe-Cr Model Alloys}

In this research, the element-resolved ferromagnetic microstructure of the two $\mathrm{Fe}-\mathrm{Cr}$ alloys was investigated using PEEM-XMCD measurements to understand the role of magnetism on the structure and on the stability of binary $\mathrm{Fe}-\mathrm{Cr}$ alloys. The magneto-optical image presented in Figure 5 shows the magnetic surface microstructure of the Fe-Cr alloy containing $12 \mathrm{wt}$ pct in Cr. A close-up (also shown in Figure 5) better visualizes the domain structure, which consists of a regular column pattern with domains magnetized parallel and antiparallel to the polarization vector of the beam. The sample exhibits microscopic magnetic domains in different magnetization directions, indicating that domains of different remanent magnetization states coexist at the surface of the alloy (identified by their gray level). By taking the difference of images recorded at resonance with right- and left-handed circularly polarized X-rays, the magnetic dichroism contrast is clearly visible. Quantitative evaluation of the spin and the orbital magnetic moments of the iron absorbing atom 


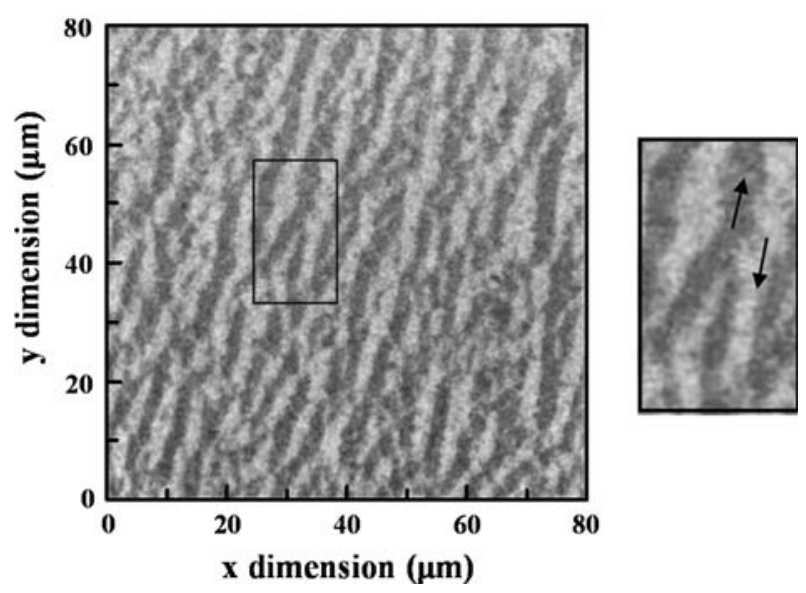

Fig. 5-Magneto-optical image recorded by means of PEEM on Fe12 pet $\mathrm{Cr}$ alloy and showing the magnetic domain patterns obtained at the $\mathrm{FeL}_{2,3}$ absorption edges. Arrows in the magnification at the right side refer to different magnetization directions.

can be determined from the absorption and XMCD spectra extracted from the PEEM images. ${ }^{[10]}$ The compositional difference of the alloys results in different size, shape, and density of the magnetic domains leading to different experimental absorption (Figure 6) and XMCD spectra (Figure 7). A multiplet structure visible at the $\mathrm{FeL}_{3}$ edge on the individual absorption spectra (Figure 6) suggests the presence of both metallic and oxide iron components. ${ }^{[1-13]}$ The peak at $708 \mathrm{eV}$ close to the iron nominal absorption energy value is attributed to metallic iron atoms. In contrast to the absorption spectra, the splitting of the $\mathrm{FeL}_{3}$ peak disappears on the individual XMCD spectra (Figure 7) of the two alloy systems leading to a single $\mathrm{L}_{3}$ peak at $708 \mathrm{eV}$, and indicates the nonferromagnetic character of the iron oxide surface layer. The amount of oxide in the alloy structure can be estimated by fitting the absorption spectrum recorded on each alloy using an atomic multiplet code. These calculations reveal that the oxide contribution is similar for the two alloy systems and represents $\sim 50$ pct of the absorption signal. An extended analysis dealing with this issue is currently performed.

A comparison of Fe- 6 pet $\mathrm{Cr}$ and $\mathrm{Fe}-12$ pet $\mathrm{Cr}$ alloys, depicted in Figure 7, shows different magnitudes in both the $L_{2}$ and $L_{3}$ peaks of the XMCD signals. This indicates different magnetic moments of iron in the alloys as a function of the alloy's composition. Therefore, the $\mathrm{Cr}$ content of the $\mathrm{Fe}-\mathrm{Cr}$ alloy influences the magnetic properties of these binary systems. These quantitative estimations of the iron magnetic moments can be directly compared to $a b$ initio calculations to obtain vital information on the magnetic state of the material and simultaneously verify modeling calculations, which are necessary for building multiscale calculations of ferritic materials for a future of predictive modeling. ${ }^{[14]}$

Complementary work to understand the influence of magnetism with alloy composition on its structure has been performed on the $\mathrm{Fe}-\mathrm{Cr}$ alloys using EXAFS to measure the variation of the local atomic structure of the $\mathrm{Fe}$ and $\mathrm{Cr}$ atoms. Information obtained on the coordination environment of $\mathrm{Cr}$ in the $\mathrm{Fe}-\mathrm{Cr}$ alloys indicates a dependence of structural ordering on magnetic ordering as a function of $\mathrm{Cr}$ content. ${ }^{[14]}$ These results lead to a deeper understanding of the role of $\mathrm{Cr}$ concentration in changing the structural and magnetic properties of $\mathrm{Fe}-\mathrm{Cr}$ alloys, which are issues of fundamental importance in materials development in $\mathrm{Fe}-\mathrm{Cr}$ alloys both in experiment and in modeling.

\section{SUMMARY}

The application of synchrotron radiation X-ray techniques shown in this research reveals their successful application to understanding the microstructural properties of materials, which is a limiting factor of their

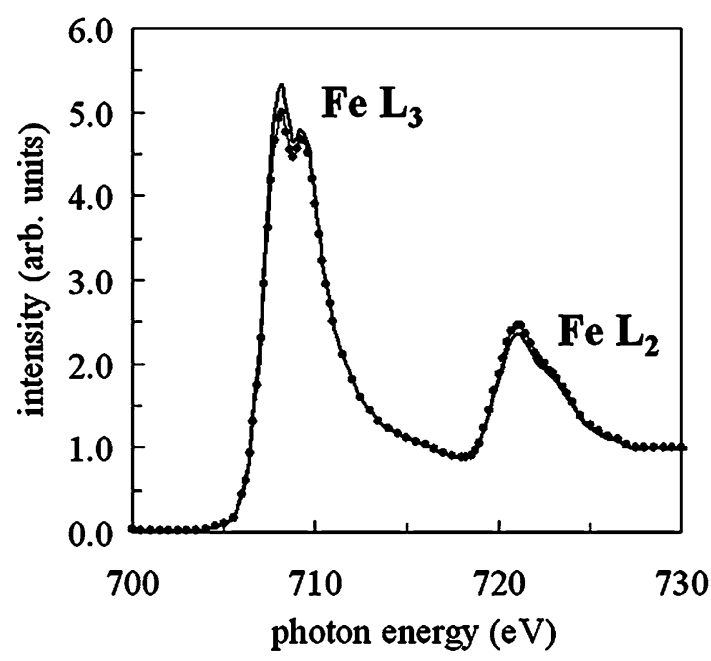

(a)

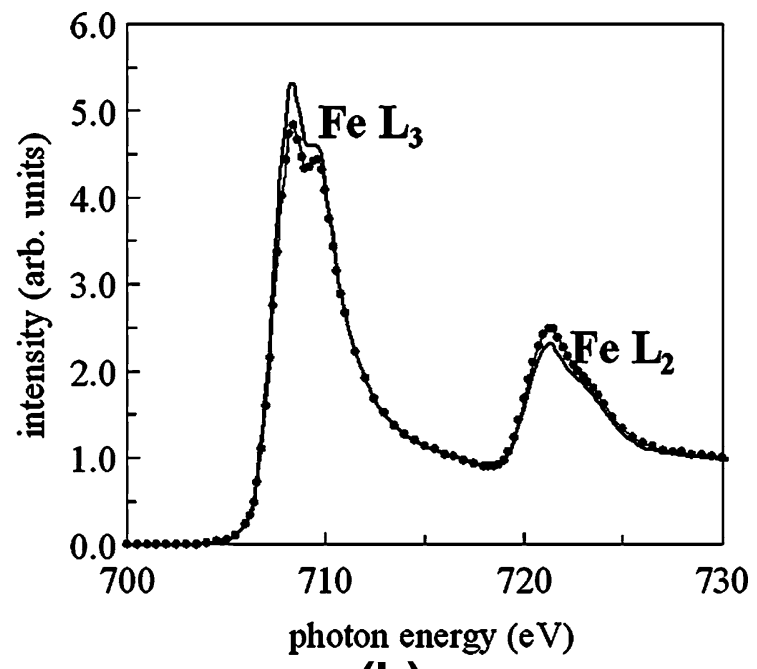

(b)

Fig. 6-Normalized X-ray absorption spectra recorded at $\mathrm{FeL}_{2,3}$ edges for opposite directions of magnetization (circle line and solid line) and fixed beam polarization helicity on $(a) \mathrm{Fe}-6$ pct $\mathrm{Cr}$ and $(b) \mathrm{Fe}-12$ pet $\mathrm{Cr}$ alloys. 


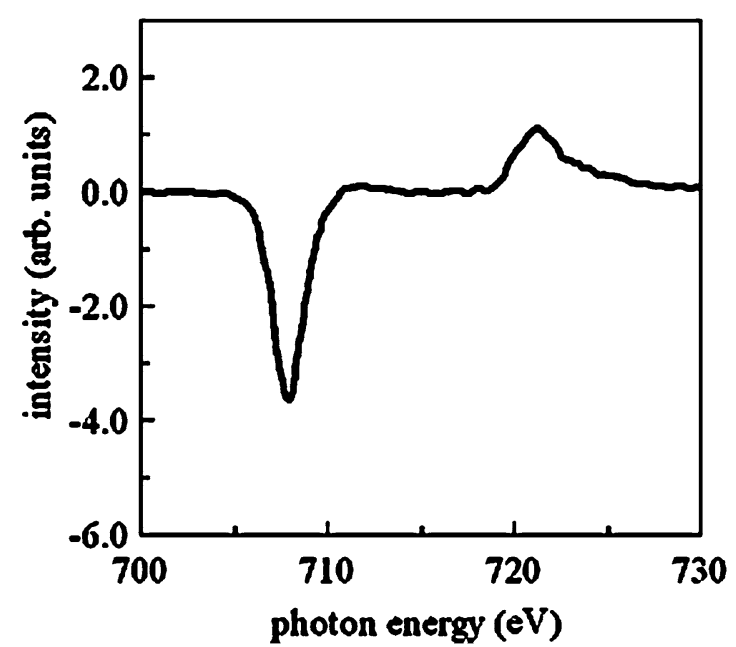

(a)

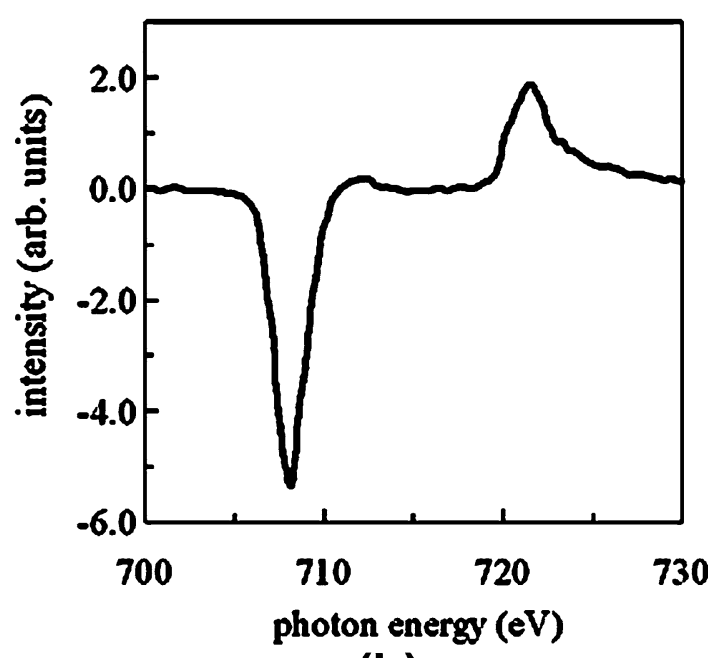

(b)

Fig. 7-Normalized XMCD spectra of $(a) \mathrm{Fe}-6$ pct $\mathrm{Cr}$ and $(b) \mathrm{Fe}-12$ pct $\mathrm{Cr}$ alloys determined by taking the difference between the absorption spectra of Figs. 6(a) and (b) and by multiplying the difference by a factor of 10 .

lifetimes, and indicates that these techniques will aid the design of candidate materials for advanced fission plants. The EXAFS measurements have been shown to be able to monitor the changes of yttria particles during irradiation, the development of nanograined structure developing in Zircaloy as a result of heavy cold deformation, and the relation of structure with magnetism. The XMCD measurements have enabled the visualization of domain structures, a quantitative estimation of spin and orbit coupling which can be used to validate modeling studies and to better understand the phase diagrams of steels. Therefore synchrotron beamline techniques emerge as powerful tools for understanding and predicting materials properties.

\section{ACKNOWLEDGMENTS}

The authors thank Professor M. Victoria and Dr. C. Degueldre for their comments and support. We also thank Drs. S. Schuppler and P. Nagel for their help at the ANKA-WERA beamline, Dr. M. Nazmy for making the $\mathrm{Fe}-\mathrm{Cr}$ alloys, A. Bullemer for the sample preparation, Dr. M. Niffenegger for the magnetization of the samples, Dr. D. Keavney for the estimation of the oxide contributions in the alloys, and Dr. F. Nolting for his help in the initial test experiments and for continual discussions. Work was performed within the Swiss Generation IV project. It was partly supported by the EU FP7 projects RAPHAEL and EXTREMAT.

\section{REFERENCES}

1. A Technology Roadmap for Generation IV Nuclear Energy Systems, http://www.ne.doe.gov/genIV/documents/gen_IV_road map.pdf, DOE, Washington, DC, 2002.

2. W. Hoffelner and M. Samaras: Modeling Advanced Materials, 2006 ANS Annual Meeting, June 4-8, 2006; J. Nucl. Mater., 2007, vol. 371 , pp. $28-36$.

3. B. Bako, D. Weygand, M. Samaras, J.-C. Chen, M. Pouchon, P. Gumbsch, and W. Hoffelner: Philos. Mag., 2007, vol. A87, pp. $3645-49$.

4. C. Degueldre, S. Conradson, and W. Hoffelner: Comput. Mater. Sci., 2005, vol. 33, pp. 3-12.

5. M.A. Pouchon, A.J. Kropf, A. Froideval, C. Degueldre, and W. Hoffelner: J. Nucl. Mater., 2007, vol. 362, pp. 253-58.

6. M.A. Pouchon, J. Chen, M. Döbeli, and W. Hoffelner: J. Nucl. Mater., 2006, vol. 352, pp. 57-61.

7. C. Degueldre, S. Conradson, A. Amato, and E. Campitelli: J. Nucl. Mater., 2006, vol. 352, pp. 126-35.

8. E. Tenckhoff: Zirconium in the Nuclear Industry 14th Int. Symp., P. Rudling and B. Kammenzind, eds., ASTM STP 1467, ASTM, Philadelphia, PA, 2006, pp. 25-50.

9. K.Y. Zhu, A. Vassel, F. Brisset, K. Lu, and J. Lu: Acta Mater., 2004, vol. 52, pp. 4101-10.

10. C.T. Chen, Y.U. Idzerda, H.-J. Lin, N.V. Smith, G. Meigs, E. Chaban, G.H. Ho, E. Pellegrin, and F. Sette: Phys. Rev. Lett., 1995, vol. 75, pp. 152-55.

11. K. Fauth, E. Goering, G. Schütz, and L.T. Kuhn: J. Appl. Phys., 2004, vol. 96, pp. 399-403.

12. E. Pellegrin, M. Hagelstein, S. Doyle, H.O. Moser, J. Fuchs, D. Vollath, S. Schuppler, M.A. James, S.S. Saxena, L. Niesen, O. Rogojanu, G.A. Sawatzky, C. Ferrero, M. Borowski, O. Tjernberg, and N.B. Brookes: Phys. Status Solidi B, 1999, vol. 215 , pp. 797-801.

13. F. Schedin, E.W. Hill, G. Van der Laan, and G. Thornton: J. Appl. Phys., 2004, vol. 96, pp. 1165-69.

14. A. Froideval, R. Iglesias, M. Samaras, S. Schuppler, P. Nagel, D.J. Keavney, M. Victoria, and W. Hoffelner: unpublished research. 